\title{
Enhancement of perpendicular magnetic anisotropy and Dzyaloshinskii-Moriya interaction in thin ferromagnetic films by atomic-scale modulation of interfaces
}

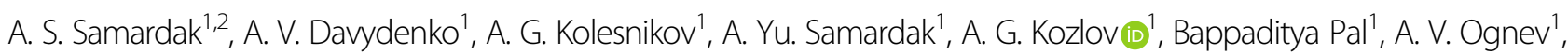 \\ A. V. Sadovnikov $\mathbb{1}^{3,4}$, S. A. Nikitov ${ }^{3,4}$, A. V. Gerasimenko ${ }^{5}$, In Ho Cha ${ }^{6}$, Yong Jin Kim , Gyu Won Kim (1) ${ }^{6}$, \\ Oleg A. Tretiakov $\mathbb{B}^{7,8}$ and Young Keun Kim $\mathbb{D}^{6}$
}

\begin{abstract}
To stabilize nontrivial spin textures, e.g., skyrmions or chiral domain walls in ultrathin magnetic films, an additional degree of freedom, such as the interfacial Dzyaloshinskii-Moriya interaction (IDMI), must be induced by the strong spin-orbit coupling (SOC) of a stacked heavy metal layer. However, advanced approaches to simultaneously control the IDMI and perpendicular magnetic anisotropy (PMA) are needed for future spin-orbitronic device implementations. Here, we show the effect of atomic-scale surface modulation on the magnetic properties and IDMI in ultrathin films composed of $5 d$ heavy metal/ferromagnet/ $4 d(5 d)$ heavy metal or oxide interfaces, such as Pt/CoFeSiB/Ru, Pt/CoFeSiB/ $\mathrm{Ta}$, and $\mathrm{Pt} / \mathrm{CoFeSiB} / \mathrm{MgO}$. The maximum IDMI value corresponds to the correlated roughness of the bottom and top interfaces of the ferromagnetic layer. The proposed approach for significant enhancement of PMA and the IDMI through interface roughness engineering at the atomic scale offers a powerful tool for the development of spinorbitronic devices with precise and reliable controllability of their functionality.
\end{abstract}

\section{Introduction}

A direct contact between a ferromagnetic metal (FM) and a heavy metal (HM) promotes fascinating spin-orbit coupling (SOC)-driven phenomena, including perpendicular magnetic anisotropy (PMA $)^{1}$, antisymmetric exchange between neighboring spins of $3 d$ metals (namely, a Dzyaloshinskii-Moriya interaction ${ }^{2-5}$ ), chiral damping of magnetic domain walls $^{6}$, topological spin textures (skyrmions $^{7,8}$, skyrmioniums ${ }^{9}$, bimerons ${ }^{10}$ ), the spin Hall effect $^{11}$, spin-orbit torque $(\mathrm{SOT})^{12,13}$, and other spinrelated effects ${ }^{14,15}$. The practical applications of these

\footnotetext{
Correspondence: A S. Samardak (samardak.as@dvfu.ru) or Young

Keun Kim (ykim97@korea.ac.kr)

${ }^{1}$ School of Natural Sciences, Far Eastern Federal University, Vladivostok 690950, Russia

${ }^{2}$ National Research South Ural State University, Chelyabinsk 454080, Russia Full list of author information is available at the end of the article
}

phenomena include the intensive development of field-free spin current-induced magnetization switching ${ }^{16,17}$, skyrmion racetrack memory ${ }^{18,19}$, SOT nanooscillators ${ }^{20}$, and neuromorphic ${ }^{21,22}$, and $\operatorname{logic}^{23}$ devices.

Recently, it has been shown that the interfacial Dzyaloshinskii-Moriya interaction ${ }^{24}$ depends not only on SOC and the lack of structural inversion symmetry but also on the degree of $3 d-5 d(4 d)$ orbital hybridization around the Fermi level ${ }^{25}$. Most of the theoretical studies presenting ab initio calculations of the spin-orbit effects assume atomically smooth interfaces and, consequently, that the IDMI is constant throughout the interface ${ }^{2,26}$. However, realistic systems have layer roughness and intermixing on the interfaces at the atomic length scale, causing fluctuations in the interaction between the localized spins and heavy metal atoms with high $\mathrm{SOC}^{27}$. As a result, the fluctuating SOC affects the IDMI, surface 
magnetic anisotropy and, consequently, dynamics of existing chiral spin textures. For the nucleation and stabilization of a spin texture such as a skyrmion, IDMI values larger than their critical values for Néel domain wall formation are required ${ }^{28}$.

To strengthen the IDMI, the structural inversion symmetry of a layered system has to be broken, and the interface quality has to tend to be an ideal surface without any disorder ${ }^{27}$. However, even for perfect epitaxially grown layers, a local (at atomic level) variation in the IDMI occurs due to lattice strain ${ }^{29}$. If one induces IDMIs of opposite sign at the top and bottom interfaces of an FM layer using different heavy metals, it is possible to significantly increase this interaction due to the additive effect $^{30}$. One promising solution is to achieve the correlated roughness of both the top and bottom interfaces, which cancels the FM layer thickness variation and, consequently, stabilizes the IDMI.

The IDMI strength is extremely sensitive to the thickness of a ferromagnetic layer $\left(t_{\mathrm{FM}}\right)^{31}$. The interface degradation at small $t_{\mathrm{FM}}$ values stimulates the nonlinear behavior of the IDMI as $1 / t_{\mathrm{FM}}$. Recently, the influence of the interface quality, considering the surface roughness and atomic intermixing, on the IDMI has been studied in symmetric $\mathrm{Pt} / \mathrm{Co} / \mathrm{Pt}$ systems ${ }^{32}$. Since identical interface qualities or ideally smooth interfaces lead to vanishing of the IDMI in symmetric systems, such as $\mathrm{Pt} / \mathrm{Co} / \mathrm{Pt}$ and $\mathrm{Ru} /$ $\mathrm{Co} / \mathrm{Ru}$, an additional treatment could be used (e.g., deposition of films at high temperatures ${ }^{32,33}$ or the introduction of an ultrathin intermediate layer of a heavy metal $^{34}$ ) to induce an interface quality asymmetry. If we assume that in layered systems with broken structural inversion symmetry ${ }^{25,30}$ the quality parameter (which can be determined as the difference between the roughness parameters, such as amplitude and period, of the top and bottom interfaces of an FM layer) goes to zero, the IDMI will be maximized. The correlated roughness of both interfaces satisfies this assumption, resulting in enhancement of PMA, as demonstrated theoretically ${ }^{35}$.

\section{Materials and methods}

\section{Sample preparation}

$\mathrm{Si}(111) \quad$ substrate $/ \mathrm{Cu}(10 \mathrm{ML}) / \mathrm{Pd}(0-56 \mathrm{ML}) \quad$ samples with roughness variation were evaporated in an ultrahigh vacuum system (Omicron Nanotechnology), consisting of a molecular beam epitaxy (MBE) chamber interconnected with an analytical chamber. $\mathrm{Si}(111)$ substrates misoriented towards [11-2] by $0.1^{\circ}$ were used. Before being loaded into the chamber, the $\mathrm{Si}(111)$ substrates were rinsed with isopropyl alcohol and distilled water. Then, the substrates were heated at $500^{\circ} \mathrm{C}$ by indirect heating for $12 \mathrm{~h}$. Immediately before deposition, the samples were flashheated by a direct current at $1200^{\circ} \mathrm{C}$ three times for $10 \mathrm{~s}$ and slowly cooled to $50^{\circ} \mathrm{C}$. All the metals were evaporated from the high-temperature effusion cells. The thickness of the layers was measured in monolayers (ML): $1 \mathrm{ML} \mathrm{Cu}$ corresponds to the thickness of one ideal layer of $\mathrm{Cu}$, which is equal to $2.09 \AA ; 1 \mathrm{ML}$ of Pd is equal to $2.25 \AA$. The growth rates of $\mathrm{Cu}$ and $\mathrm{Pd}$ were 4.3 and $0.75 \mathrm{ML} / \mathrm{min}$, respectively. The temperature of the substrate was $75^{\circ} \mathrm{C}$ during both $\mathrm{Cu}$ and $\mathrm{Pd}$ deposition. To prevent intermixing of $\mathrm{Pd}$ and $\mathrm{Si}$ and to initiate the epitaxial growth of $f c c$ $\mathrm{Pd}(111)$, a $\mathrm{Cu}(10 \mathrm{ML})$ buffer layer was formed on the $\mathrm{Si}$ surface before the deposition of the Pd buffer layer. The lattice period and structure of $\mathrm{Pd}$ during growth were analyzed by employing reflection high-energy electron diffraction (RHEED, Staib Instruments). The RHEED measurements were performed simultaneously with the deposition of the samples. The topography of the $\mathrm{Pd}$ buffer layer was investigated in situ using a scanning tunneling microscope manufactured by Omicron Nanotechnology. The Pd surface roughness was defined ex situ on areas of $5 \times 5 \mu \mathrm{m}^{2}$ and $2 \times 2 \mu \mathrm{m}^{2}$ using atomic force microscopy (AFM, Ntegra Aura, NT-MDT). Immediately after the MBE process, the following three series of samples were deposited on the top of the Pd surface by magnetron sputtering: $\mathrm{MgO}$-series, $\mathrm{Pt}(2) / \mathrm{CoFeSiB}(1.5) /$ $\mathrm{MgO}(2) / \mathrm{Ta}(5)$; Ta-series, $\mathrm{Pt}(2) / \mathrm{CoFeSiB}(1.5) / \mathrm{Ta}(5)$; and $\mathrm{Ru}$-series, $\mathrm{Pt}(2) / \mathrm{CoFeSiB}(1.5) / \mathrm{Ru}(3) / \mathrm{Ta}(5)$, where the layer thickness is indicated in $\mathrm{nm}$, as shown in Supplementary Fig. S1. The ferromagnetic layer has the composition $\mathrm{Co}_{70.5} \mathrm{Fe}_{4.5} \mathrm{Si}_{15} \mathrm{~B}_{10}$, represented in at. ${ }^{36}$.

\section{Atomic structure observation}

Scanning transmission electron microscopy (STEM) images and electron energy loss spectra (EELS) were collected using a Cs-corrected Titan 80-300 microscope operated at $300 \mathrm{kV}$ and equipped with a Gatan Quantum 966 spectrometer. The convergence and collection angles were 24.9 and $24.7 \mathrm{mrad}$, respectively. TEM sample preparation was performed in a precision ion-polishing system (PIPS, Gatan model 691) with an ion beam energy of $3.5 \mathrm{keV}$ and a milling angle of $6^{\circ}$ in double sector milling mode. To prevent a temperature increase, the sample was cooled to $-165^{\circ} \mathrm{C}$ using liquid nitrogen during milling. To characterize the elemental depth profile, secondary ion mass spectrometry (SIMS, TOF. SIMS 5) using $\mathrm{Cs}^{+}$ions was utilized.

\section{Surface and interface roughness measurements}

The surface morphology was studied using STM and AFM (Ntegra Aura, NT-MDT). To analyze the crystal structure and interface quality (roughness, intermixing, and thickness variation), we used analytical methods including $\mathrm{X}$-ray diffraction (XRD) and X-ray reflectivity (XRR). The study was performed on a SmartLab (Rigaku) X-ray diffractometer with $\mathrm{CuK \alpha}$ radiation $(1.54 \AA)$. Simulations of the XRR spectra were performed with GlobalFit software. 


\section{Magnetic characterization}

The magnetic properties of the films were investigated in terms of the magneto-optical Kerr effect (NanoMOKE II, Durham Magneto Optics) by using a vibrating sample magnetometer (7410 VSM, LakeShore). Magnetic force microscopy (Ntegra Aura, NT-MDT) was used to observe the magnetic domain structure in an applied magnetic field using low magnetic moment tips. Magnetization reversal processes were studied by a Kerr microscope (Evico Magnetics).

\section{Results and discussion}

In this study, we artificially introduced interface modulation using an atomically smooth $\mathrm{Si}(111) / \mathrm{Cu}$ surface with an epitaxially grown Pd seed layer of various nominal thicknesses $\left(t_{\mathrm{Pd}}\right)$ ranging from 0 to $12.6 \mathrm{~nm}$, as shown in Fig. 1. One of the advantages is that an epitaxial Pd seed layer, independent of its thickness and in the absence of crystal grains, affects the degree of crystallinity and the interface quality of the consequently grown layer of $\mathrm{Pt}$. The root-mean-square $(\mathrm{rms})$ roughness $\left(R_{q}\right)$ depends on $t_{\mathrm{Pd}}$ and varies from 0.15 to $1.0 \mathrm{~nm}$, which corresponds to atomic-scale modulation of the surface. First, multilayer structures with periodic subnanometer interface modulation were fabricated by a continuous hybrid growth process using a sequence of molecular beam epitaxy (MBE) for the formation of a Pd buffer layer with the desired nanoscale surface pattern and magnetron sputtering for deposition of an $\mathrm{HM}_{1} / \mathrm{FM} / \mathrm{HM}_{2}$ (oxide) layered system with enhanced PMA and IDMI functionality. As a result, we prepared three series of samples, each of which consisted of a 1.5-nm-thick ferromagnetic $\mathrm{CoFeSiB}$ layer asymmetrically sandwiched between one $5 d \mathrm{HM}(\mathrm{Pt})$ layer and one $\mathrm{MgO}$ layer (namely, the MgO-series), two $5 d \mathrm{HM}$ layers (Pt and Ta) (namely, the Ta-series), or one $5 d \mathrm{HM}$ layer $(\mathrm{Pt})$ on the bottom and one $4 d \mathrm{HM}$ layer $(\mathrm{Ru})$ on the top (namely, the Ru-series). The layering scheme of the experimental samples is shown in Supplementary Fig. S1. We report the direct dependence of the IDMI magnitude on the quality of the interfaces. We find that the morphological correlation of the top and bottom interfaces at $t_{\mathrm{Pd}}=10.35 \mathrm{~nm}$ results in the peak values of the IDMI for the three series of samples.

Pd island growth leads to a significant increase in $R_{q}$ as well as the average amplitude $\left(R_{a}\right)$ and period $\left(P_{a}\right)$ of the Pd surface roughness (see Fig. $1 \mathrm{~g}$ ). The persistent increase in the Pd surface roughness is caused by three different growth modes revealed by scanning tunneling microscopy (STM) (the corresponding images are shown in Fig. 1a-d), as indicated in Fig. 1g, and supported by reflective high-energy electron diffraction (RHEED) ${ }^{37,38}$ : (i) $\mathrm{Pd}$ grows in 2D mode from the beginning and up to $0.6 \mathrm{~nm}$ (Fig. 1a), (ii) layer-by-layer growth mode takes place from 0.6 to $2.9 \mathrm{~nm}$ (Fig. 1b), and (iii) 3D island growth occurs at $t_{\text {Pd }}$ values larger than $2.9 \mathrm{~nm}$ (Fig. 1c, d). As a result, we fabricated epitaxial samples with periodically modulated Pd surfaces with average amplitudes and periods of isotropic longwave roughness ranging from 0.2 to $1.5 \mathrm{~nm}$ and 0.75 to $57.0 \mathrm{~nm}$, respectively.

The RHEED pattern of the Pd seed layer with $t_{\mathrm{Pd}}=$ $1.125 \mathrm{~nm}$ had streaks, and almost no spots are visible, which indicates reflection diffraction (see insets in Fig. 1b). If there were transmission diffraction, one would see a two-dimensional ordered lattice without curvature, which forms when the three-dimensional inverse lattice is crossed by the Ewald sphere (it can be approximated by a plane due to its large radius). However, as the thickness of the Pd layer increases, spots appear on the streaks as a consequence of the transmission diffraction, which is manifested by developed surface topography and confirmed by probe methods. It can also be noted that the RHEED background level for the Pd layer with $t_{\mathrm{Pd}}=$ $1.125 \mathrm{~nm}$ is slightly higher than that for other thicknesses, which indicates an increased content of defects at the initial stages of growth. The background did not change in the other samples (Fig. 1c, d), meaning that the quality of the Pd crystal structure remains constant.

Subsequent magnetron sputtering allowed us to grow three series of samples with controllable roughness of each interface, as shown in Fig. $1 \mathrm{~h}-\mathrm{j}$. The roughness data for the Pd and Ta layers were defined experimentally by STM and AFM, respectively. The layer roughness of the top interfaces of $\mathrm{Pt}, \mathrm{CoFeSiB}, \mathrm{MgO}$, and $\mathrm{Ru}$ was determined by fitting the X-ray reflectivity (XRR) data. We recorded the XRR spectra for incident X-ray beam angles $(\theta)$ ranging from $0^{\circ}$ to $6^{\circ}$. An example of an XRR spectrum is shown in Supplementary Fig. S2. After performing the fitting procedure by means of GlobalFit software, we found the best agreement between the experimental and calculated reflexivity curves. As seen in Fig. $1 \mathrm{~h}-\mathrm{j}$, the $R_{\mathrm{q}}$ values of $\mathrm{Pd}, \mathrm{Pt}, \mathrm{CoFeSiB}$, and the capping layer converge to a point at $t_{\mathrm{Pd}}=10.35 \mathrm{~nm}$ for all three series, indicating the correlation of the interfaces.

Analysis of the island profiles (Fig. 1e, f) and crosssectional high-angle annular dark-field scanning transmission electron microscopy (HAADF-STEM) and highresolution transmission electron microscopy (HRTEM) images (Fig. 2a) enabled the identification of each layer and its crystal structure. The high sensitivity to atomic number contrast HAADF-STEM imaging allowed us to distinguish different layers and to determine their thicknesses, which agreed well with the designated growth parameters. HRTEM imaging in combination with $2 \mathrm{D}$ fast Fourier transform (2D FFT) analysis confirmed the epitaxial growth of $f c c(111)$-Pd (Fig. 2b). The observed shortwave roughness induced by the atomic steps was smoothed out by the subsequent magnetron sputtering of 


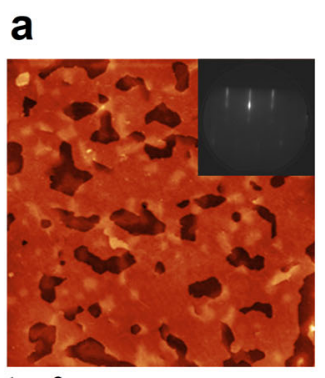

$\mathbf{t}_{\mathrm{Pd}}=\mathbf{0} \mathbf{n m}$

e
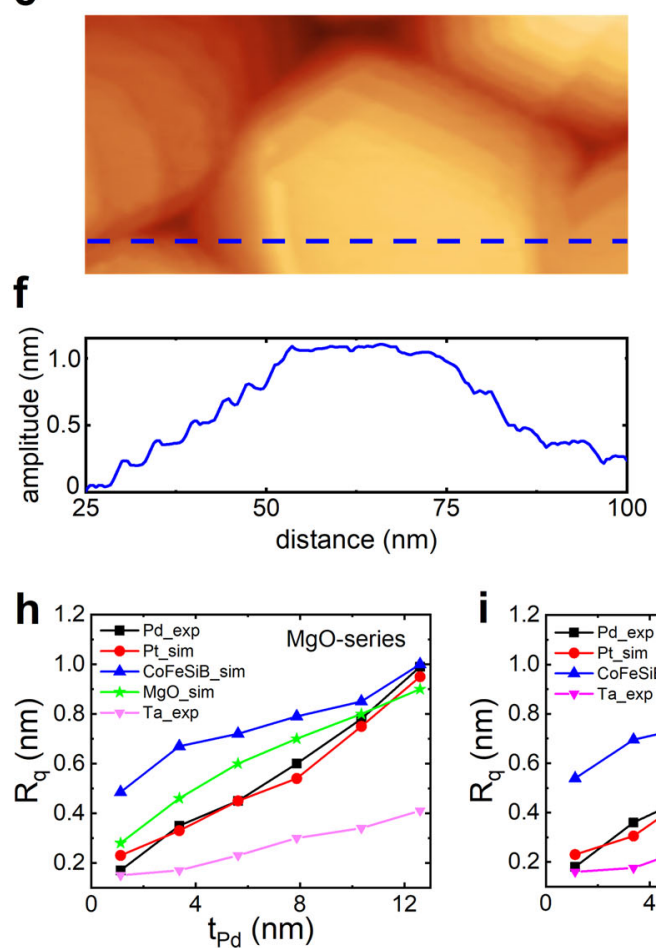

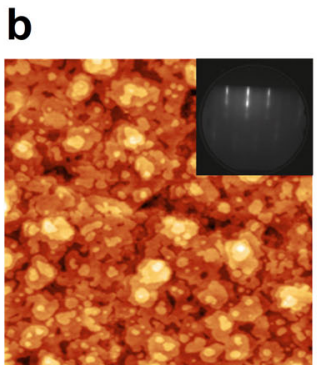

$1.13 \mathrm{~nm}$

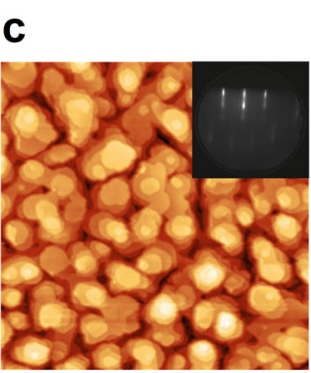

$3.38 \mathrm{~nm}$

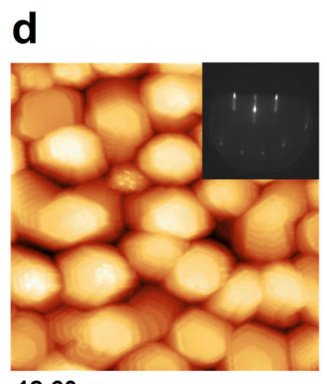

$12.60 \mathrm{~nm}$

g
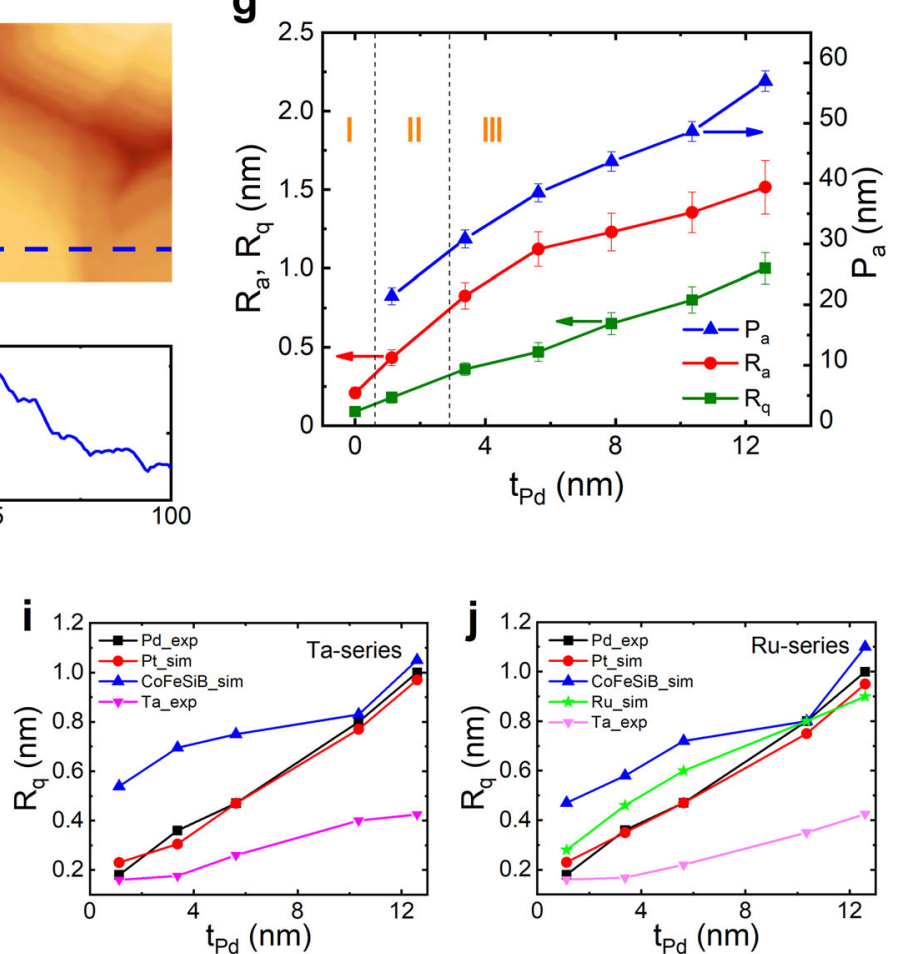

Fig. 1 Surface images and roughness parameters of various film structures characterized by STM, AFM, and XRR. a-d The 200 $\times 200 \mathrm{~nm}^{2}$ STM images of $\mathrm{Si}(111) / \mathrm{Cu}(2.1) / \mathrm{Pd}$ surfaces of various thicknesses. Insets show RHEED patterns for corresponding samples. e Magnified STM image of a Pd island grown during the 12.6-nm-thick Pd layer deposition, and $\mathbf{f}$ its corresponding $x$-profile. $\mathbf{g}$ Dependence of the average amplitude of roughness $\left(R_{a}\right)$, root-mean-square roughness $\left(R_{q}\right)$, and average period of roughness $\left(P_{a}\right)$ on the nominal thickness of the Pd buffer layer ( $\left.t_{\mathrm{Pd}}\right)$. Root-mean-square roughness $\left(R_{q}\right)$ of layers of the $(\mathbf{h}) \mathrm{MgO}-, \mathbf{i}$ Ta-, and $\mathbf{j}$ Ru-series as a function of $t_{\mathrm{Pd}}$. Experimental values were measured by STM and AFM. Simulation values were calculated from the fitting of the experimental XRR spectra.

a 2-nm thick Pt layer. As the lattice mismatch between Pd and $\mathrm{Pt}$ was in the range from 1 to $8 \%$ depending on the Pd lattice parameter ${ }^{38}$, the Pt layer had the crystal structure of Pd: it grew with the same $f c c(111)$ texture and repeated the Pd surface modulation, as shown in Fig. $2 \mathrm{a}$, c. The formed $\mathrm{Pd}(111) / \mathrm{Pt}(111)$ stack can be considered a coherent system or a superlattice because these two metals have the same crystal phase and a small lattice mismatch. Surprisingly, CoFeSiB, which usually has an amorphous structure ${ }^{36}$, possessed crystallinity with an $f c c$-like lattice and repeated the surface morphology of the $\mathrm{Pd} / \mathrm{Pt}$ stack, demonstrating practically quasi-epitaxial growth. The subsequent $\mathrm{Ru}$ and partial Ta layers had a pronounced $f c c(111)$ crystalline structure, as indicated by the 2D FFT patterns in Fig. 2a. The electron energy loss spectroscopy (EELS) elemental profile is presented in the left panel of Fig. 2a to show the distribution of Pd, Co, and $\mathrm{Ru}$ atoms in the layers. The upper part of the Ta layer was oxidized to a depth of $3.5 \mathrm{~nm}$ from the top. Secondary-ion mass spectrometry (SIMS) (Fig. 2d) and energy-dispersive X-ray spectroscopy (EDX) (Fig. 2e-g) proved the intermixing on interfaces and boron diffusion. The Pd layer-induced surface curvature of the correlated interfaces is visible in the magnified HAADF-STEM 
a
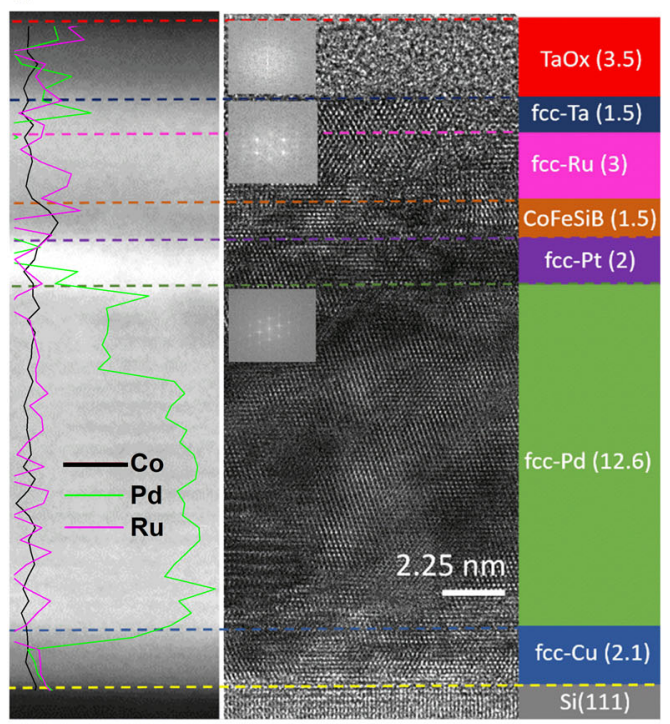

C

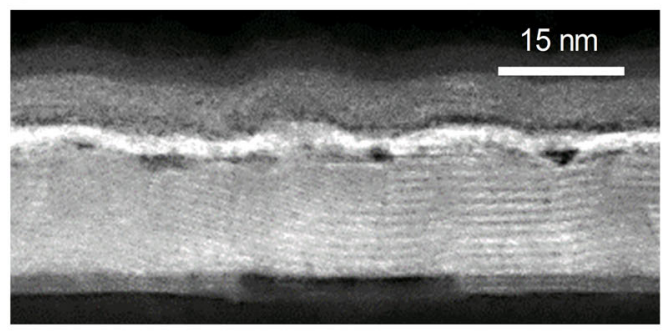

e

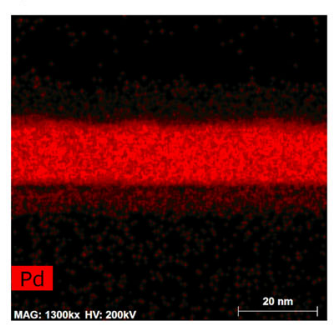

f

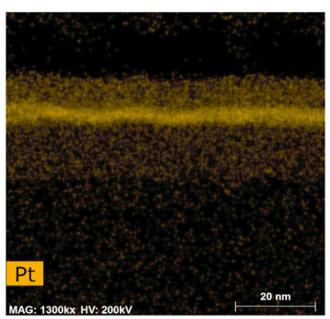

b

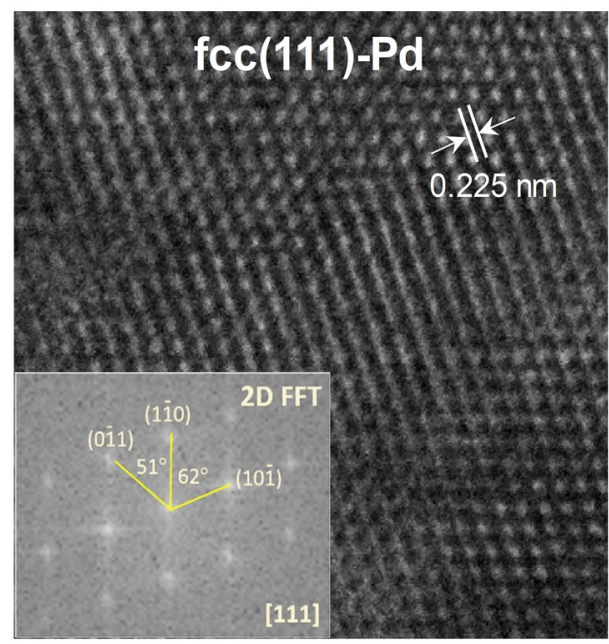

d

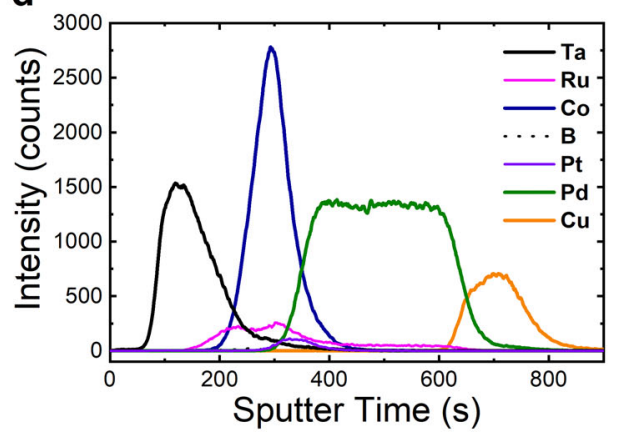

g

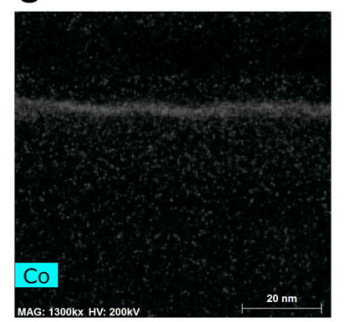

h

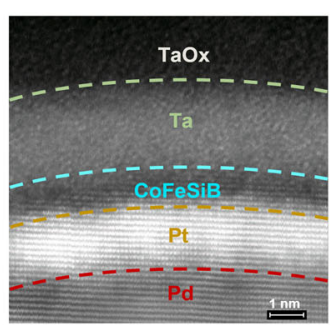

Fig. 2 Microstructure and elemental distribution characterized by TEM and SIMS. a Cross-sectional HAADF-STEM with EELS elemental analysis (left) and HRTEM (right) images of the $\mathrm{Si}(111) / \mathrm{Cu}(2.1) / \mathrm{Pd}(12.6) / \mathrm{Pt}(2) / \mathrm{CoFeSiB}(1.5) / \mathrm{Ru}(3) / \mathrm{Ta}(5)$ sample. The insets in the HRTEM image consist of 2D FFT patterns for corresponding areas. b Atomic-resolution imaging of the Pd layer and corresponding 2D FFT pattern supporting fcc(111)-Pd phase formation. c Large-area cross-sectional HAADF-STEM image enabling observation of the surface modulation. $\mathbf{d}$ Original SIMS spectrum of the designated element distribution for the presented sample. Note that boron (B) intensity is too low to be recognized in the original ion counts. e- $\mathbf{g}$ EDS elemental maps for the $\mathrm{Si}(111) / \mathrm{Cu}(2.1) / \mathrm{Pd}(12.6) / \mathrm{Pt}(2) / \mathrm{CoFeSiB}(1.5)$ sample. $\mathbf{h}$ The magnified high-resolution HAADF-STEM image with the visible curvature of correlated interfaces. The dashed lines are shown for guidance only.

image of the $\mathrm{Si}(111) / \mathrm{Cu}(2.1) / \mathrm{Pd}(12.6) / \mathrm{Pt}(2) / \mathrm{CoFeSiB}$ (1.5)/Ta sample (Fig. 2h).

It is noteworthy that the samples from the Ta-series sputtered on the Pd layer with thicknesses ranging from 1.125 to $12.6 \mathrm{~nm}$ had fourfold out-of-plane anisotropy with easy axes tilted at $\sim 45^{\circ}\left(225^{\circ}\right)$ relative to the sample plane (see Supplementary Fig. S3). Samples from the Ruseries had in-plane magnetic anisotropy only, with the disorientation of the easy crystallographic axes within the range of $-30^{\circ}$ to $30^{\circ}$ relative to the sample plane; see Supplementary Fig. S4. Excluding only the very thin Pd layer, all as-deposited samples from the MgO-series had 

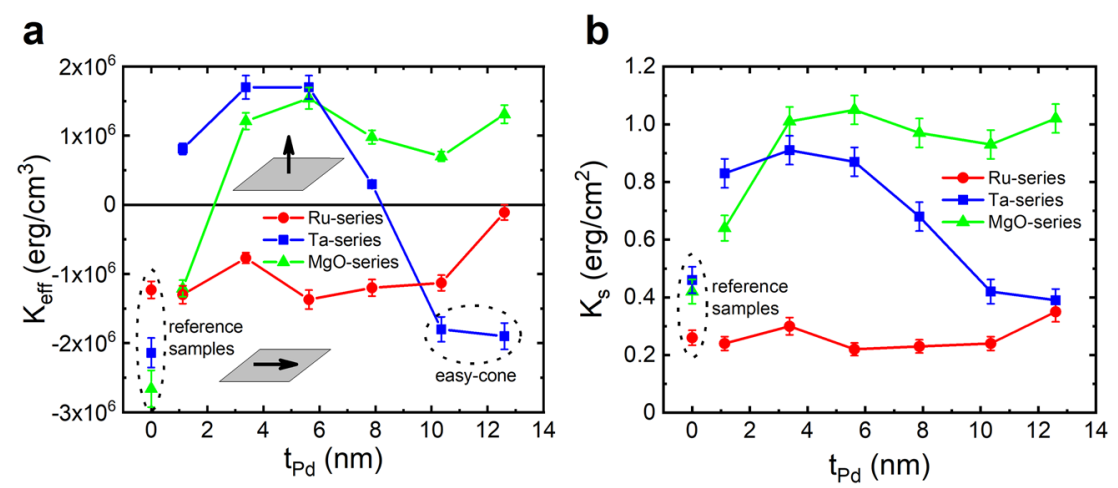

Fig. 3 Pd thickness-dependent variation of magnetic anisotropies. a Effective magnetic anisotropy energy and $\mathbf{b}$ surface anisotropy energy as a function of $t_{\mathrm{Pd}}$ for the three series of samples.

PMA (see Supplementary Fig. S5). These results were supported by the values of the effective magnetic anisotropy $K_{\text {eff }}$, as shown in Fig. 3a. The anisotropy energy of the magnetic system can be described as $E(\theta)=-2 \pi\left(M_{s}^{\text {eff }}\right)^{2} \sin ^{2} \theta+K_{1} \sin ^{2} \theta+K_{2} \sin ^{4} \theta$, where $\theta$ is the polar angle of magnetization, $M_{s}^{\text {eff }}$ is the effective saturation magnetization of an FM layer with the effective thickness $t_{\mathrm{eff}}$, and $K_{1}$ and $K_{2}$ are the first-order and second-order anisotropy constants, respectively. The first-order effective magnetic anisotropy is defined as $K_{\text {eff }}=K_{1}-2 \pi\left(M_{s}^{\text {eff }}\right)^{2}$, where $K_{1}=\frac{2 K_{s}}{t_{\text {eff }}}$, and $K_{s}$ is the surface anisotropy energy. Samples with $t_{\mathrm{Pd}}=10.35$ and $12.6 \mathrm{~nm}$ from the Ta-series existed in the easy-cone state $^{39,40}$, the formation of which requires the implementation of the following conditions: $K_{\text {eff }}<0, K_{2}>0$, and $K_{2}>-\frac{1}{2} K_{\text {eff }}$. Since we know the cone state angle $\theta=45^{\circ}$, the $K_{2}$ value can be calculated using the relation $\sin \theta=\sqrt{\frac{-K_{\text {eff }}}{2 K_{2}}}$ with the solution $K_{2}=-K_{\text {eff. }}$ The $K_{\text {eff }}$ values were calculated by fitting the Brillouin light scattering (BLS) spectra (which are discussed below), providing that the values of $M_{s}^{\text {eff }}$ were defined from the linear slopes of $M_{s} t_{\mathrm{FM}}=f\left(t_{\mathrm{FM}}\right)$ dependences ${ }^{41}$, where $t_{\mathrm{FM}}$ is the nominal thickness of the FM layer, as shown in Fig. 3a.

The observed magnetic anisotropy has two main contributions: one arising from spin-orbit (SO) effects and resulting in PMA and the other arising from long-range dipolar interactions in the presence of randomly periodic magnetic surfaces of high curvature. The SO-induced anisotropy may be enhanced by increased electron scattering when the interface becomes rougher, which is consistent with the $t_{\mathrm{Pd}}$ dependence shown in Fig. 3. In the case of magnetic anisotropy due to dipolar interactions, the magnetic interface curvature corresponding to the observed samples should lead to an easy-cone anisotropy $^{19}$, which is strikingly confirmed by the abovementioned measurements. More specifically, the approximation of surface morphology for samples with $t_{\mathrm{Pd}}=3.38$ and $12.6 \mathrm{~nm}$ gave a sinusoidal function with parameters (see Supplementary Section 4, Fig. S6) that, as calculated according to the method developed in Ref. ${ }^{19}$, lead to easy-cone anisotropy. As shown in Fig. 3b, the main contribution to PMA of the MgO- and Ta-series was surface anisotropy, while in the $\mathrm{Ru}$-series, the surface contribution was not sufficient to compete with the demagnetizing energy.

The direct measurement of the IDMI was performed by BLS spectroscopy based on the IDMI-driven asymmetric dispersion shift of long-wavelength thermal spin waves in Damon-Eshbach surface mode ${ }^{42,43}$. The effective IDMI energy density $\left(D_{\text {eff }}\right)$ was determined from the direct measurements of the frequency shift $(\Delta f)$ between Stokes and anti-Stokes spin-wave propagation regimes, $f_{s}$, and $f_{\text {as }}$, respectively:

$$
\Delta_{f}=f_{s}-f_{\mathrm{as}}=2 \gamma D_{\mathrm{eff}} k / \pi M_{s},
$$

where $k$ is the magnon wavevector and $\gamma=176 \mathrm{GHz} / \mathrm{T}$ is the gyromagnetic ratio for $\mathrm{CoFeB}^{44}$. The effective saturation magnetization $M_{s}$ was measured to be 935 , 953, and $704 \mathrm{emu} / \mathrm{cm}^{3}$ for the MgO-, Ta- and Ru-series, respectively, which is in good agreement with the experimental data. Examples of the normalized Stokes and anti-Stokes spectra for three series of samples can be seen in Fig. 4a-c. The corresponding field and wavevector dependences of peak frequency shifts are shown in Fig. $4 \mathrm{~d}-\mathrm{f}$. In the case of surface spin-wave modes (the Damon-Eshbach geometry) in ultrathin magnetic films, spin waves are localized on the top or bottom interfaces depending on the direction of the wave vector $k$. If these interfaces have different surface anisotropies $\left(K_{S 1}\right.$ and $K_{S 2}$ are the surface anisotropies of the bottom and top interfaces, respectively), then the spin waves are affected by different effective fields on the top and bottom 

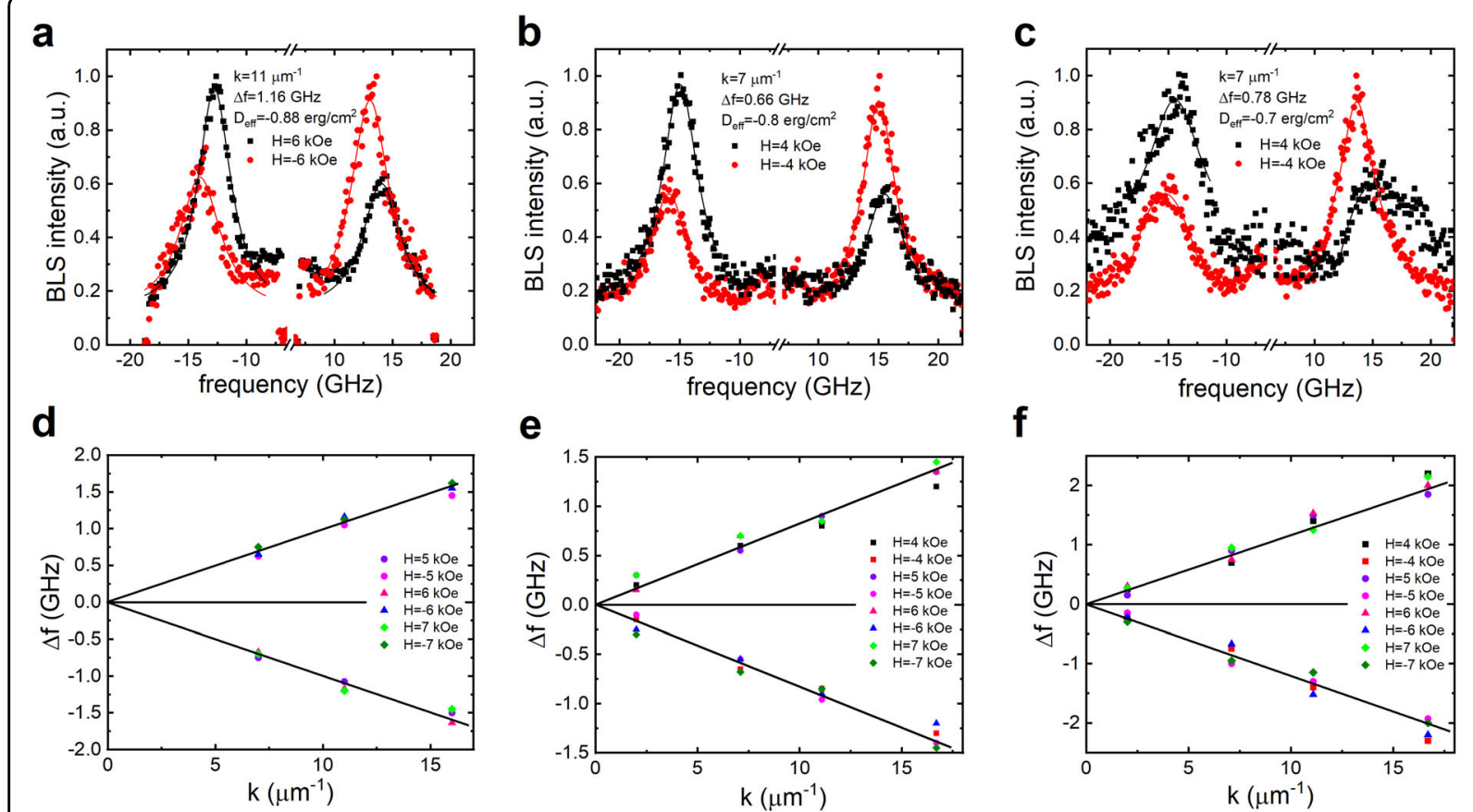

e

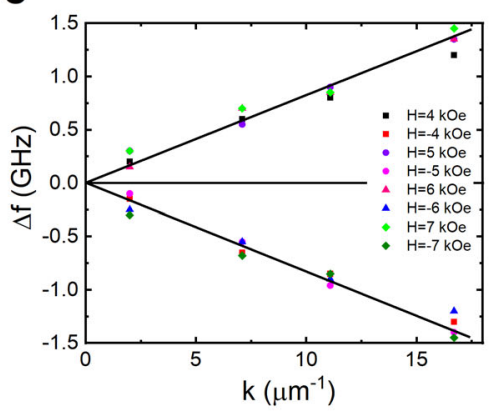

f
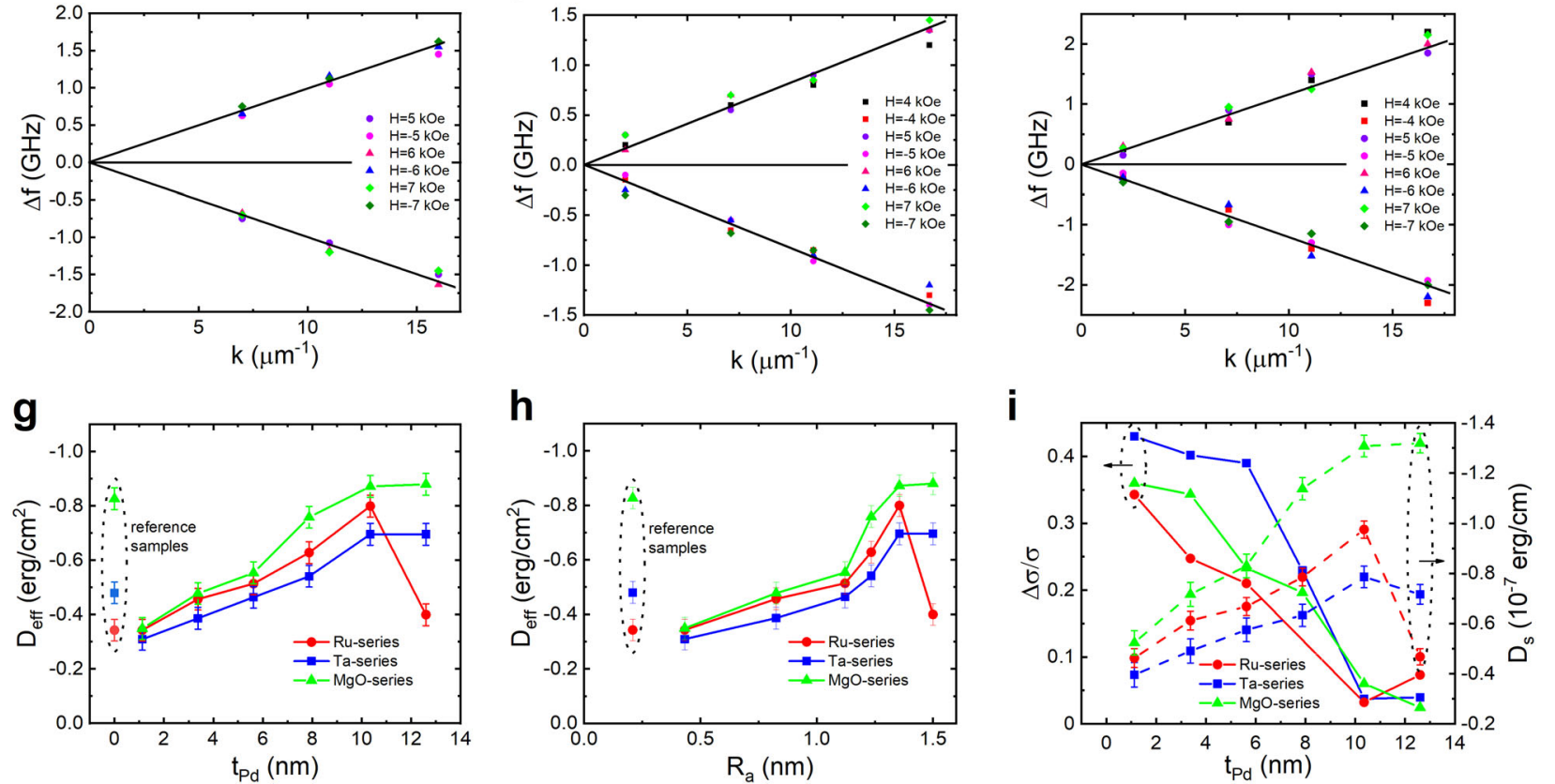

Fig. 4 BLS spectra and effective IDMI values depending on the Pd thickness. High-resolution BLS spectra and corresponding dependences of peak frequency shifts $\Delta f$ on the wave vector $k$ measured in positive and negative fields for films with $t_{\mathrm{Pd}}=10.35 \mathrm{~nm}$ taken from the $(\mathbf{a}, \mathbf{d}) \mathrm{MgO}-,(\mathbf{b}, \mathbf{e})$ Ta-, and $(\mathbf{c}, \mathbf{f})$ Ru-series. The solid lines reflect the fit of the experimental data. Dependences of $D_{\text {eff }}$ on the nominal thickness of the Pd layer $(\mathbf{g})$ and on the amplitude of roughness $R_{a}(\mathbf{h})$ for MgO-, Ta- and Ru-series. (i) The interface quality factor $\Delta \sigma / \sigma$ and surface IDMl constant $\left(D_{s}\right)$ depending on $t_{\mathrm{Pd}}$ for the three series of samples.

interfaces, which in turn lead to different frequencies of the Stokes and anti-Stokes peaks. Thus, the difference in surface anisotropy can contribute to $\Delta f$. To separate the contributions from the IDMI and $K_{S}$, it is necessary to evaluate the influence of $K_{S 1}$ and $K_{S 2}$ on $\Delta f$. To do this, we used an analytical model of dipole-exchange spin waves ${ }^{45}$, in the framework of which the frequency shift due to $K_{S}$ can be calculated as:

$$
\Delta f_{k}=\frac{8 \gamma}{\pi^{2}} \frac{K_{S 1}-K_{S 2}}{M_{S}} \frac{k}{1+\frac{l_{e x}^{2} \pi^{2}}{t_{F M}^{2}}},
$$

where $l_{e x}=\sqrt{\frac{2 A}{4 \pi M_{S}^{2}}}$ is the exchange length. This equation is an approximation for thin ferromagnetic films $\left(k t_{\mathrm{FM}} \ll 1\right)$, as in the case of our experiments. The calculation of the surface anisotropy effect gives values of $\Delta f<50 \mathrm{MHz}$ (this value is comparable with the BLS measurement error), while the experimentally observed $\Delta f$ values can achieve $1.6 \mathrm{GHz}$. This fact allows us to conclude that the IDMI is the main source of the frequency shift in our systems. Moreover, the observed linear dependence of $\Delta f$ on the wave vector $k$ proves that the frequency shift is due to the anti-symmetrical exchange induced by the IDMI $^{31,45}$.

As the reference samples for the MgO-, $\mathrm{Ta}$ - and $\mathrm{Ru}$ series, we used $\mathrm{Si} / \mathrm{SiO}_{2} / \mathrm{Pt}(2) / \mathrm{CoFeSiB}(1.5) / \mathrm{MgO}(1) / \mathrm{Ta}$ (5), $\mathrm{Si} / \mathrm{SiO}_{2} / \mathrm{Pt}(2) / \mathrm{CoFeSiB}(1.5) / \mathrm{Ta}(5)$, and $\mathrm{Si} / \mathrm{SiO}_{2} / \mathrm{Pt}(2) /$ $\mathrm{CoFeSiB}(1.5) / \mathrm{Ru}(3) / \mathrm{Ta}$ (5) films, respectively. All these asdeposited films had in-plane anisotropy only and minimal 
surface roughness. Recently, it has been shown that Pt, Ta, and $\mathrm{Ru}$ have a negative IDMI at the bottom interface, leading to partial cancellation of the effective IDMI $\left(D_{\text {eff }}\right)$ if a ferromagnet layer is sandwiched between them ${ }^{46,47}$. We defined by BLS that these three reference samples have negative effective values of the IDMI: $D_{\text {eff }}=-0.83$, -0.48 , and $-0.34 \mathrm{erg} / \mathrm{cm}^{2}$, respectively. If we assume that the $\mathrm{CoFeSiB} / \mathrm{MgO}$ interface had zero IDMI, then the $\mathrm{CoFeSiB} / \mathrm{Ta}$ and $\mathrm{CoFeSiB} / \mathrm{Ru}$ interfaces would possess positive IDMI with approximate values of 0.35 and $0.49 \mathrm{erg} / \mathrm{cm}^{2}$, respectively. This result proves the partial compensation effect of the IDMI induced at the bottom and top interfaces in a $\mathrm{Pt}\left(D_{\text {bottom }}<0\right) / \mathrm{CoFeSiB} / \mathrm{Ru}$ or Ta $\left(D_{\text {top }}>0\right)$ structure.

The increasing thickness of the Pd seed layer leads to significant changes in the IDMI compared to the reference samples, as shown in Fig. 4g. The sputtering of the corresponding layer sequence on the $\mathrm{Si}(111) / \mathrm{Cu}(2.1) / \mathrm{Pd}$ surface with $t_{\mathrm{Pd}}=1.125 \mathrm{~nm}$ sharply decreased the $D_{\text {eff }}$ to $-0.33 \pm 0.04 \mathrm{erg} / \mathrm{cm}^{2}$ for all the series of samples. The subsequent increase in $t_{\mathrm{Pd}}$ to $10.35 \mathrm{~nm}$ leads to a significant increase in $D_{\text {eff }}$ (see Fig. $4 \mathrm{~g}$ ). We excluded the effect of the degree of crystallinity and size of $\mathrm{Pt}(111)$ grains emerging in polycrystalline or textured systems, which may change with the Pd seed layer thickness, on the strength of the exchange interactions at the interface ${ }^{48}$ because the grown $\mathrm{Pd}(111) / \mathrm{Pt}(111)$ stacks have an epitaxial structure without the formation of crystallites, as supported by the complex HAADF-STEM, HRTEM, RHEED, and X-ray diffraction analyses; the XRD spectra and their descriptions are shown in Supplementary Fig. S7. Thus, our findings show that the small variation in the periodic surface roughness with an amplitude $<1.0 \mathrm{~nm}$ resulted in $\mathrm{Pt} / \mathrm{CoFeSiB}$ interface modulation at the atomic scale, which was sufficient for a drastic enhancement of the IDMI value. In the $\mathrm{Ru}$-series at $t_{\mathrm{Pd}}>10.35 \mathrm{~nm}$, the effective IDMI rapidly decreased to $-0.4 \mathrm{erg} / \mathrm{cm}^{2}$, while it remained constant for the $\mathrm{MgO}$ - and Ta-series. The dependence of $D_{\text {eff }}$ on the amplitude of roughness $R_{a}$ is demonstrated in Fig. 4h.

IDMI enhancement mainly arises from the increased electron scattering at the interfaces in the presence of a strong spin-orbit interaction, in the same way spin-orbit torques are enhanced ${ }^{46}$. Based on previous theoretical work, it is expected that disorder may further increase the Dzyaloshinskii-Moriya interaction strength since it increases the scattering of electrons. The latter, in the presence of an emergent magnetic field due to a spin-orbit interaction, gives rise to spin-dependent scattering, thus leading to additional spin accumulation at the interface with the ferromagnet. This accumulation of spins, in turn, may exert a torque on the magnetization ${ }^{49}$. Therefore, at highly disordered interfaces such as those studied here, the effects due to spin-orbit coupling, such as the IDMI, are expected to be enhanced. The dependence of the IDMI and the spin Hall effect on disorder strength may be probed based on the nonequilibrium Green function in the Keldysh formalism ${ }^{50}$. This scattering is increased when the interfacial surface becomes less smooth, especially when intermixing is present. The overall increase in the effective interfacial surface contributes to this enhancement as well.

Thus, we showed the correlation between the Pd layer thickness (or surface roughness) and the magnitude of the effective IDMI. However, to find a direct correlation, one must analyze the roughness and intermixing on $\mathrm{Pt} /$ $\mathrm{CoFeSiB}$ and $\mathrm{CoFeSiB} / \mathrm{MgO}(\mathrm{Ta}, \mathrm{Ru})$ interfaces. A crosssectional HRTEM observation does not allow direct inspection of interfacial intermixing. To qualitatively analyze the layer compositions and possible intermixing, we employed SIMS using $\mathrm{Cs}^{+}$ions (an example is shown in Fig. 2d). For all the series of samples, we found that the intermixing on the $\mathrm{Pt} / \mathrm{CoFeSiB}$ interface did not depend on the Pd layer thickness but maintained a constant depth. The intermixing depth of the top $\mathrm{CoFeSiB} / \mathrm{MgO}$ interface was practically zero, which is in agreement with previous studies ${ }^{51-53}$. In contrast, the top $\mathrm{CoFeSiB} / \mathrm{Ta}$ and $\mathrm{CoFeSiB} / \mathrm{Ru}$ interfaces demonstrated an increased intermixing depth with increasing $t_{\mathrm{Pd}}$. The most reliable quantitative method for this estimation is XRR, which enabled us to extract the $R_{q}$ value (Fig. $1 \mathrm{~h}-\mathrm{j}$ ) and average intermixing depth $\left(I_{d}\right)$ for each interface. This approach gives a set of parameters of the multilayer structure, including surface/interface roughness, layer thickness, and density variation due to the intermixing ${ }^{54}$.

To analyze the quality of interfaces, we introduced the quality factor $\Delta \sigma / \sigma$, where $\Delta \sigma$ is the difference between the roughness values of the top and bottom interfaces of the CoFeSiB layer $\left(R_{q}^{\text {top }}-R_{q}{ }^{\text {bottom }}\right)$ and $\sigma$ is the sum of roughness values $\left(R_{q}^{\text {top }}+R_{q}^{\text {bottom }}\right)$. The dependence of $R_{q}$ on the top interfaces of all the layers with the Pd thickness is represented in Fig. $1 \mathrm{~h}-\mathrm{j}$. The main trend was an increase in the interface roughness for all the layers with increasing $t_{\mathrm{Pd}}$. Notably, the initial $R_{q}$ for $\mathrm{CoFeSiB}$ was higher than that for the other layers. This could be related to the initially amorphous nature of this ferromagnetic material. With an increase in $t_{\mathrm{Pd}}$ to $10.35 \mathrm{~nm}$, the roughness of the CoFeSiB layer approached the $R_{q}$ of the lower Pt layer, which corresponds to the roughness correlation of the top and bottom interfaces. The experimental data revealed that the Ta capping layer smoothed out the surface roughness. Based on these data, we plotted the dependence $\Delta \sigma / \sigma=f\left(t_{\mathrm{Pd}}\right)$ for the Ru- and Ta-series, as shown in Fig. 4i. One can see the correlation between the interface quality factor and the surface IDMI constant $\left(D_{s}\right)$ for both series. The $D_{s}$ constant is the thicknessindependent indicator of the IDMI strength and can be defined as $D_{s}=D_{\text {eff }} t_{\text {eff }}$, where $t_{\text {eff }}$ is the effective thickness 
of a ferromagnetic layer considering the magnetically dead layer (MDL) thickness $(\Delta)$ of the top and bottom interfaces $\left(\Delta=\Delta_{\text {top }}+\Delta_{\text {bottom }}\right)$. From the XRR analysis, we found that the intermixing depth $\left(I_{d}\right)$ on the Pt/CoFeSiB interface was the same for samples from the three series $\left(I_{d}=0.24 \mathrm{~nm}\right)$, and it did not depend on $t_{\mathrm{Pd}}$. According to our investigation and studies conducted by different groups, $\Delta_{\text {bottom }}$ for a Pt/FM interface is zero or even negative due to the effect of the proximity-induced magnetization $^{55}$. However, the top $\mathrm{CoFeSiB} / \mathrm{MgO}$, $\mathrm{CoFeSiB} / \mathrm{Ta}$, and $\mathrm{CoFeSiB} / \mathrm{Ru}$ interfaces had different values of $I_{d}$ : This parameter was zero for $\mathrm{CoFeSiB} / \mathrm{MgO}$, it increased from 0.22 to $0.47 \mathrm{~nm}$ with an increase in $t_{\mathrm{Pd}}$ for $\mathrm{CoFeSiB} / \mathrm{Ta}$, and it increased from $0.15 \mathrm{~nm}$ to $0.33 \mathrm{~nm}$ with an increase in $t_{\mathrm{Pd}}$ from 1.125 to $12.6 \mathrm{~nm}$ for $\mathrm{CoFeSiB} / \mathrm{Ru}$. The $I_{d}$ was largest for the $\mathrm{CoFeSiB} / \mathrm{Ta}$ interface because there was more intense interdiffusion between layers. In addition, these findings can be explained in terms of interfacial enthalpy, which is a source of interdiffusion, and its values vary for different materials ${ }^{51,56}$. Since intermixing is the main reason for MDL formation ${ }^{57,58}$, we suggest that $I_{d}$ coincides with the $\Delta_{\text {top }}$ for the CoFeSiB/Ta and CoFeSiB/Ru interfaces. The defined MDL depths for $\mathrm{Ta}$ and $\mathrm{Ru}$ are in good agreement with the data found for the $\mathrm{CoFeB} / \mathrm{Ta}(\mathrm{Ru})$ interfaces ${ }^{53,59,60}$. Finally, one can find that $D_{s}=D_{\text {eff }}\left(t_{\mathrm{FM}}-\Delta_{t o p}\right)$. This formula was used to calculate $D_{s}$ as subject to $t_{\mathrm{Pd}}$, as shown in Fig. $4 \mathrm{i}$.

With a small Pd roughness, the magnitude of $\Delta \sigma / \sigma$ is maximal, which corresponds to the highly uncorrelated top and bottom interfaces of the CoFeSiB layer, leading to relatively small $D_{s}$ values. The flowing tendency of $\Delta \sigma / \sigma$ with increasing $t_{\mathrm{Pd}}$ reflects the growing correlation of the interfaces, causing a significant increase in $D_{s}$ of approximately twofold for the three series of samples. The same increase in $D_{s}$ continuing up to $t_{\mathrm{Pd}}=10.35 \mathrm{~nm}$ suggests the same nature of this effect in all the series of samples. The further increase in $t_{\mathrm{Pd}}$ and, consequently, its $R_{q}$, whose value is consistent with the CoFeSiB thickness, is accomplished by the decrease in $D_{\mathrm{s}}$ for the $\mathrm{Ru}$ - and Taseries. The maximum value of $D_{s}=-1.1 \times 10^{-7} \mathrm{erg} / \mathrm{cm}$ was found for the $\mathrm{Pt} / \mathrm{CoFeSiB} / \mathrm{MgO}$ system, which is comparable to those of other Co-based systems such as $\mathrm{Pt} / \mathrm{Co}_{2} \mathrm{FeAl}_{0.5} \mathrm{Si}_{0.5} / \mathrm{MgO}\left(D_{s}=-0.42 \times 10^{-7} \mathrm{erg} / \mathrm{cm}\right.$, measured by BLS ${ }^{61}, \mathrm{Pt} / \mathrm{Co} / \mathrm{AlO}_{\mathrm{x}}\left(D_{s}=-1.7 \times 10^{-7} \mathrm{erg} / \mathrm{cm}^{62}\right.$ and $1.4 \times 10^{-7} \mathrm{erg} / \mathrm{cm}^{63}$, measured by $\left.\mathrm{BLS}\right), \mathrm{Pt} / \mathrm{CoFeB} /$ $\mathrm{MgO}$ and $\mathrm{Pt} / \mathrm{CoFe} / \mathrm{MgO}\left(D_{s}=0.8 \times 10^{-7} \mathrm{erg} / \mathrm{cm}^{64}\right.$ and $-1.27 \times 10^{-7} \mathrm{erg} / \mathrm{cm}^{65}$, measured by $\left.\mathrm{BLS}\right), \mathrm{Pt} / \mathrm{Co} / \mathrm{MgO}$ $\left(D_{s}=2.17 \times 10^{-7} \mathrm{erg} / \mathrm{cm} \text {, measured by } \mathrm{BLS}\right)^{26}, \mathrm{Pt} / \mathrm{Co} / \mathrm{Ir} /$ Pt $\left(D_{s}=0.84 \times 10^{-7} \mathrm{erg} / \mathrm{cm}\right.$, measured by the asymmetric magnetic domain growth $)^{34}$, and $\mathrm{Ta} / \mathrm{Pt} / \mathrm{Co} / \mathrm{Ir}\left(D_{s}=1.6 \times\right.$ $10^{-7} \mathrm{erg} / \mathrm{cm}$ and $2.2 \times 10^{-7} \mathrm{erg} / \mathrm{cm}$, measured by BLS and the asymmetric hysteresis, respectively) ${ }^{66}$. Notably, in most of the papers, the magnetically dead layer depth is not taken into account for the calculation of $D_{s}$, resulting in overestimated values. As shown, the magnitudes of the IDMI are particularly similar for the same systems. Furthermore, the signs of the IDMI, even for the same IDMI measurement method, are opposite. Such discrepancy for the BLS results may be a product of an incorrect definition of the Stokes and anti-Stokes peaks relative to the applied in-plane magnetic field. A case in which a Stokes peak has a larger intensity than an anti-Stokes peak has to be considered as measured at the positive magnetic field and vice versa; see Fig. $4 \mathrm{a}-\mathrm{c}$. Then, if the Stokes frequency $\left(f_{s}\right)$ is smaller (larger) than anti-Stokes frequency $\left(f_{\text {as }}\right)$, the IDMI value will be negative (positive) since $D_{s} \sim\left|f_{s}\right|-f_{\text {as }}{ }^{42}$. Based on this fact, our analysis of the works representing the IDMI data measured by BLS revealed that, in contrast to the reported data ${ }^{26,31,64-66}$, the sign of $D_{s}$ for $\mathrm{Pt} / \mathrm{Co}$ based systems is negative, promoting the formation of left-handed cycloidal spin structures ${ }^{2}$.

The observed effect of the periodic surface modulation on the IDMI can be explained within a model of correlated roughness of interfaces, where the amplitude and period of roughness are the same for both the top and bottom interfaces of the $\mathrm{CoFeSiB}$ layer and the parameter $\Delta \sigma / \sigma$ goes to zero. In this case, the thickness of $\mathrm{CoFeSiB}$ remains unchanged, giving, as a result, a value near the maximum IDMI value, which must be similar for ideally smooth interfaces. For the uncorrelated roughness regime, the period of roughness is the same, but the roughness amplitude for the top and bottom interfaces is different $\left(R_{q}^{\text {top }}>R_{q}^{\text {bottom }}\right)$. In this case, $\Delta \sigma / \sigma \gg 0$, which means there are local variations in the CoFeSiB layer thickness and, consequently, local variations in the IDMI.

\section{Conclusion}

In this article, the roughness-dependent enhancement of the IDMI has been successfully demonstrated for the first time. We have shown that the correlated roughness of the top and bottom interfaces can increase the IDMI values by up to 2.5 times, with the maximum observed value being $D_{s}=-1.1 \times 10^{-7} \mathrm{erg} / \mathrm{cm}$, which is the largest known IDMI for CoFeB-based systems. The main reasons for this enhancement are the intermixing at the bottom and top interfaces and the correlated interface-roughness variations, which can both affect electronic transport across the interface and, as a result, the degree of electron scattering. The latter is the main driver of the IDMI and other spin-orbit effects ${ }^{49}$.

\section{Acknowledgements}

This research was supported by the Future Materials Discovery Program through the National Research Foundation of Korea funded by the Ministry of Science and ICT (2015M3D1A1070465), by the Samsung Electronics University R\&D program (Q1514435), by the Russian Foundation for Basic Research (1902-00530), by the Russian Ministry of Science and Higher Education under the state task (0657-2020-0013), and by Act 211 of the Government of the Russian Federation (No. 02.A03.21.0011). O.A.T. acknowledges the support by the 
Australian Research Council (Grant No. DP200101027), the Cooperative Research Project Program at the Research Institute of Electrical Communication, Tohoku University, and by the Ministry of Science and Technology Higher Education of the Russian Federation in the framework of Increase Competitiveness Program of NUST "MISiS" (No. K2-2019-006), implemented by a governmental decree dated 16th of March 2013, N 211. A.V. S. and S.A.N. thank the Russian Foundation for Basic Research (18-29-27026 and 18-57-76001).

\section{Author details}

'School of Natural Sciences, Far Eastern Federal University, Vladivostok 690950, Russia. ${ }^{2}$ National Research South Ural State University, Chelyabinsk 454080, Russia. " ${ }^{3}$ Laboratory "Metamaterials", Saratov State University, Saratov 410012 , Russia. "Kotel'nikov Institute of Radioengineering and Electronics, Russian Academy of Sciences, Moscow 125009, Russia. Institute of Chemistry, Far East Branch, Russian Academy of Sciences, Vladivostok 690022, Russia. ${ }^{6}$ Department of Materials Science and Engineering, Korea University, Seoul 02841, South Korea. ${ }^{7}$ School of Physics, The University of New South Wales, Sydney 2052 NSW, Australia. " National University of Science and Technology "MISiS", Moscow 119049, Russia

\section{Author contributions}

A.S.S., A.V.O., O.A.T, and Y.K.K. generated the idea of the surface roughness effect and proposed the project. A.V.D., A.G.K., I.H.C., and Y.J.K. prepared the experimental samples. B.P. and A.Yu.S. measured the magnetic properties. A.V.S. and S.A.N. performed BLS spectroscopy. A.V.G. carried out the XRR and XRD studies. A.G.K. performed the micromagnetic simulations and analyzed the results with the assistance of O.A.T. The interpretation of the experimental data was performed by A.S.S. and O.A.T. with the assistance of A.V.O. A.S.S. analyzed the experimental data and wrote the majority of the manuscript with the help of O.A.T. and Y.K.K.

\section{Conflict of interest}

The authors declare that they have no conflict of interest.

\section{Publisher's note}

Springer Nature remains neutral with regard to jurisdictional claims in published maps and institutional affiliations.

Supplementary information is available for this paper at https://doi.org/ 10.1038/s41427-020-0232-9.

Received: 17 January 2020 Revised: 4 April 2020 Accepted: 18 May 2020. Published online: 17 July 2020

\section{References}

1. Hellman, F. et al. Interface-induced phenomena in magnetism. Rev. Mod. Phys. 89, 025006 (2017)

2. Yang, H. X., Thiaville, A., Rohart, S., Fert, A. \& Chshiev, M. Anatomy of Dzyaloshinskii-Moriya interaction at Co/Pt interfaces. Phys. Rev. Lett. 115, 267210 (2015).

3. Dzyaloshinsky, I. A thermodynamic theory of weak ferromagnetism of antiferromagnetics. J. Phys. Chem. Solids 4, 241-255 (1958).

4. Moriya, T. Anisotropic superexchange interaction and weak ferromagnetism. Phys. Rev. 120, 91-98 (1960).

5. Fert, A. \& Levy, P. M. Role of Anisotropic exchange interactions in determining the properties of spin-glasses. Phys. Rev. Lett. 44, 1538-1541 (1980).

6. Jué, E. et al. Chiral damping of magnetic domain walls. Nat. Mater. 15, 272 (2015).

7. Fert, A., Reyren, N. \& Cros, V. Magnetic skyrmions: advances in physics and potential applications. Nat. Rev. Mater. 2, 17031 (2017).

8. Jiang, W. et al. Skyrmions in magnetic multilayers. Phys. Rep. 704, 1-49 (2017).

9. Kolesnikov, A. G., Stebliy, M. E., Samardak, A. S. \& Ognev, A. V. Skyrmionium-high velocity without the skyrmion Hall effect. Sci. Rep. 8 , 16966 (2017)

10. Göbel, B., Mook, A., Henk, J., Mertig, I. \& Tretiakov, O. A. Magnetic bimerons as skyrmion analogues in in-plane magnets. Phys. Rev. B. 99, 060407 (2019).
11. Sinova, J., Valenzuela, S. O., Wunderlich, J., Back, C. H. \& Jungwirth, T. Spin Hall effects. Rev. Mod. Phys. 87, 1213-1260 (2015).

12. Gambardella, P. \& Miron, I. M. Current-induced spin-orbit torques. Philos. Trans. R. Soc. A 369, 3175-3197 (2011).

13. Manchon, A. et al. Current-induced spin-orbit torques in ferromagnetic and antiferromagnetic systems. Rev. Mod. Phys. 91, 035004 (2019).

14. Manchon, A. \& Belabbes A., in Solid State Physics 68 (ed. Camley, R. E. \& Stamps, R. L.) Ch.1, 1-89 (Elsevier, Cambridge, MA, USA, 2017).

15. Hoffmann, A. \& Bader, S. D. Opportunities at the Frontiers of Spintronics. Phys. Rev. Appl. 4, 047001 (2015).

16. Liu, Y., Zhou, B. \& Zhu, J.-G. Field-free magnetization switching by utilizing the spin hall effect and interlayer exchange coupling of iridium. Sci. Rep. 9, 325 (2019).

17. $\mathrm{Yu}$, G. et al. Switching of perpendicular magnetization by spin-orbit torques in the absence of external magnetic fields. Nat. Nanotechnol. 9, 548 (2014).

18. Fert, A., Cros, V. \& Sampaio, J. Skyrmions on the track. Nat. Nanotechnol. 8, 152 (2013).

19. Bessarab, P. F. et al. Lifetime of racetrack skyrmions. Sci. Rep. 8, 3433 (2018).

20. Haidar, M. et al. A single layer spin-orbit torque nano-oscillator. Nat. Commun. 10, 2362 (2019).

21. Li, S. et al. Emerging neuromorphic computing paradigms exploring magnetic skyrmions. Proc. 2018 IEEE Computer Society Annual Symposium on VLSI (ISVLSI) 539-544 (2018).

22. Prychynenko, D. et al. Magnetic skyrmion as a nonlinear resistive element: a potential building block for reservoir computing. Phys. Rev. Appl. 9, 014034 (2018).

23. Lin, X., Yang, W., Wang, K. L. \& Zhao, W. Two-dimensional spintronics for lowpower electronics. Nat. Electron. 2, 274-283 (2019).

24. Park, Y.-K. et al. Experimental observation of the correlation between the interfacial Dzyaloshinskii-Moriya interaction and work function in metallic magnetic trilayers. NPG Asia Mater. 10, 995-1001 (2018).

25. Belabbes, A., Bihlmayer, G., Bechstedt, F., Blugel, S. \& Manchon, A. Hund's ruledriven dzyaloshinskii-moriya interaction at 3d-5d interfaces. Phys. Rev. Lett. 117, 2472 (2016). (24).

26. Boulle, O. et al. Room-temperature chiral magnetic skyrmions in ultrathin magnetic nanostructures. Nat. Nanotechnol. 11, 449-454 (2016).

27. Zimmermann, B. et al. Dzyaloshinskii-Moriya interaction at disordered interfaces from ab initio theory: Robustness against intermixing and tunability through dusting. Appl. Phys. Lett. 113, 232403 (2018).

28. Thiaville, A., Rohart, S., Jué, É., Cros, V. \& Fert, A. Dynamics of Dzyaloshinskii domain walls in ultrathin magnetic films. EPL-Europhys. Lett. 100, 57002 (2012).

29. Davydenko, A. V. et al. Dzyaloshinskii-Moriya interaction in symmetric epitaxial $[\mathrm{Co} / \mathrm{Pd}(111)] \mathrm{N}$ superlattices with different numbers of Co/Pd bilayers. Phys. Rev. B. 99, 014433 (2019).

30. Moreau-Luchaire, C. et al. Additive interfacial chiral interaction in multilayers for stabilization of small individual skyrmions at room temperature. Nat. Nanotechnol. 11, 444-448 (2016).

31. Cho, J. et al. Thickness dependence of the interfacial Dzyaloshinskii-Moriya interaction in inversion symmetry broken systems. Nat. Commun. 6, 7635 (2015).

32. Wells, A. W. J., Shepley, P. M., Marrows, C. H. \& Moore, T. A. Effect of interfacial intermixing on the Dzyaloshinskii-Moriya interaction in Pt/Co/Pt. Phys. Rev. B. 95, 054428 (2017).

33. Quinsat, M. et al. Dzyaloshinskii-Moriya interaction in $\mathrm{Pt} / \mathrm{Co} / \mathrm{Pt}$ films prepared by chemical vapor deposition with various substrate temperatures. AIP Adv. 7 . 056318 (2017).

34. Hrabec, A. et al. Measuring and tailoring the Dzyaloshinskii-Moriya interaction in perpendicularly magnetized thin films. Phys. Rev. B. 90, 020402 (2014).

35. Tretiakov, O. A., Morini, M., Vasylkevych, S. \& Slastikov, V. Engineering curvatureinduced anisotropy in thin ferromagnetic films. Phys. Rev. Lett. 119, 077203 (2017).

36. Chun, B. S. et al. Effects of Co addition on microstructure and magnetic properties of ferromagnetic CoFeSiB alloy films. Acta Mater. 58, 2836-2842 (2010).

37. Davydenko, A. V. et al. Origin of perpendicular magnetic anisotropy in epitaxial $\mathrm{Pd} / \mathrm{Co} / \mathrm{Pd}(111)$ trilayers. Phys. Rev. B. 95, 064430 (2017).

38. Davydenko, A. V., Kozlov, A. G., Ognev, A. V., Stebliy, M. E. \& Chebotkevich, L. A. Structure and roughness analysis of thin epitaxial Pd films grown on $\mathrm{Cu} / \mathrm{Si}$ (111) surface. Appl. Surf. Sci. 384, 406-412 (2016).

39. Shaw, J. M. et al. Perpendicular magnetic anisotropy and easy cone state in $\mathrm{Ta} /$ Co60Fe20B20/MgO. IEEE Magn. Lett. 6, 3500404 (2015). 
40. Jang, P.-H., Oh, S.-H., Kim, S. K. \& Lee, K.-J. Domain wall dynamics in easy-cone magnets. Phys. Rev. B. 99, 024424 (2019).

41. Kim, G. W. et al. Role of the heavy metal's crystal phase in oscillations of perpendicular magnetic anisotropy and the interfacial DzyaloshinskiiMoriya interaction in W/CoFeB/MgO Films. Phys. Rev. Appl. 9, 064005 (2018).

42. Moon, J. H. et al. Spin-wave propagation in the presence of interfacial Dzyaloshinskii-Moriya interaction. Phys. Rev. B. 88, 184404 (2013).

43. Zakeri, K. Probing of the interfacial Heisenberg and Dzyaloshinskii-Moriya exchange interaction by magnon spectroscopy. J. Phys.: Condens. Matter 29 013001 (2017).

44. Rana, B., Fukuma, Y., Miura, K., Takahashi, H. \& Otani, Y. Effect of excitation power on voltage induced local magnetization dynamics in an ultrathin CoFeB film. Sci. Rep. 7, 2318 (2017).

45. Gladii, O., Haidar, M., Henry, Y., Kostylev, M. \& Bailleul, M. Frequency nonreciprocity of surface spin wave in permalloy thin films. Phys. Rev. B. 93, 054430 (2016).

46. Woo, S. et al. Observation of room-temperature magnetic skyrmions and their current-driven dynamics in ultrathin metallic ferromagnets. Nat. Mater. 15, 501-506 (2016)

47. Samardak, A. et al. Enhanced interfacial Dzyaloshinskii-Moriya interaction and isolated skyrmions in the inversion-symmetry-broken Ru/Co/W/Ru films. Appl. Phys. Lett. 112, 192406 (2018).

48. Kurt, H., Venkatesan, M. \& Coey, J. M. D. Enhanced perpendicular magnetic anisotropy in Co/Ni multilayers with a thin seed layer. J. Appl. Phys. 108, $073916(2010)$

49. Ado, I. A., Tretiakov, O. A. \& Titov, M. Microscopic theory of spin-orbit torques in two dimensions. Phys. Rev. B. 95, 094401 (2017)

50. Ado, I. A., Qaiumzadeh, A., Duine, R. A., Brataas, A. \& Titov, M. Asymmetric and symmetric exchange in a generalized 2D Rashba ferromagnet. Phys. Rev. Lett. 121, 086802 (2018).

51. Watanabe, K. et al. Annealing temperature dependence of magnetic properties of $\mathrm{CoFeB} / \mathrm{MgO}$ stacks on different buffer layers. Jpn. J. Appl. Phys. 56, 0802B2 (2017)

52. An, G.-G. et al. Highly stable perpendicular magnetic anisotropies of CoFeB/ MgO frames employing W buffer and capping layers. Acta Mater. 87, 259-265 (2015).
53. Jang, S. Y., Lim, S. H. \& Lee, S. R. Magnetic dead layer in amorphous CoFeB layers with various top and bottom structures. J. Appl. Phys. 107, 09C707 (2010).

54. Yasaka, M. X-ray thin-film measurement techniques $\mathrm{V}$. X-ray reflectivity measurement. Rigaku J. 26, 1-9 (2010).

55. Ueno, $\mathrm{T}$. et al. Enhanced orbital magnetic moments in magnetic heterostructures with interface perpendicular magnetic anisotropy. Sci. Rep. 5, 14858 (2015).

56. De Boer, F. R. in Cohesion in metals 1st edn (eds De Boer, F. R., Mattens, W. C. M., Boom, R., Miedema, A. R. \& Niessen, A. K.) (Elsevier Scientific Pub. Co, Amsterdam, Netherlands, 1988)

57. Izquierdo, J., Robles, R., Vega, A., Talanana, M. \& Demangeat, C. Origin of dead magnetic Fe overlayers on V(110). Phys. Rev. B. 64, 060404 (2001).

58. Sinha, J. et al. Enhanced interface perpendicular magnetic anisotropy in $\mathrm{Ta}$ CoFeB|MgO using nitrogen doped Ta underlayers. Appl. Phys. Lett. 102, 242405 (2013).

59. Jang, S. Y., You, C.-Y., Lim, S. H. \& Lee, S. R. Annealing effects on the magnetic dead layer and saturation magnetization in unit structures relevant to a synthetic ferrimagnetic free structure. J. Appl. Phys. 109, 013901 (2011).

60. Frankowski, M. et al. Buffer influence on magnetic dead layer, critical current, and thermal stability in magnetic tunnel junctions with perpendicular magnetic anisotropy. J. Appl. Phys. 117, 223908 (2015).

61. Belmeguenai, M. et al. Characterization of the interfacial Dzyaloshinskii-Moriya interaction in Pt/Co2FeAl0.5Si0.5 ultrathin films by brillouin light scattering. IEEE Trans. Magn. 53, 1000405 (2017).

62. Belmeguenai, M. et al. Interfacial Dzyaloshinskii-Moriya interaction in perpendicularly magnetized Pt/CO/AlO ultrathin films measured by Brillouin light spectroscopy. Phys. Rev. B. 91, 180405 (2015).

63. Kim, N.-H. et al. Improvement of the interfacial Dzyaloshinskii-Moriya interaction by introducing a Ta buffer layer. Appl. Phys. Lett. 107, 142408 (2015).

64. Di, K. et al. Asymmetric spin-wave dispersion due to Dzyaloshinskii-Moriya interaction in an ultrathin Pt/CoFeB film. Appl. Phys. Lett. 106, 052403 (2015).

65. Belmeguenai, M. et al. Brillouin light scattering investigation of the thickness dependence of Dzyaloshinskii-Moriya interaction in Co0.5Fe0.5 ultrathin films. Phys. Rev. B. 93, 174407 (2016).

66. Han, D.-S. et al. Asymmetric hysteresis for probing Dzyaloshinskii-Moriya interaction. Nano Lett. 16, 4438-4446 (2016). 